
\title{
28 Research Square \\ Prediction of Treatment Failure and Compliance in Patients with Tuberculosis
}

\section{Hyeon-Kyoung Koo}

Inje University College of Medicine https://orcid.org/0000-0002-1200-9892

Jinsoo Min

Daejeon Saint Mary's Hospital

\section{Hyung Woo Kim}

Catholic University of Korea Incheon Saint Mary's Hospital

Joosun Lee

Korea Centers for Disease Control and Prevention

\section{Ju Sang Kim}

Catholic University of Korea Incheon Saint Mary's Hospital

Jae Seuk Park

Dankook University College of Medicine

Sung-Soon Lee ( $\sim$ Sungsoonlee@gmail.com)

Inje University College of Medicine https://orcid.org/0000-0001-6422-2313

\section{Research article}

Keywords: public-private sector partnership, treatment failure, tuberculosis, Korea

Posted Date: August 19th, 2020

DOl: https://doi.org/10.21203/rs.3.rs-27791/v3

License: (c) (1) This work is licensed under a Creative Commons Attribution 4.0 International License. Read Full License

Version of Record: A version of this preprint was published on August 24th, 2020. See the published version at https://doi.org/10.1186/s12879-020-05350-7. 


\section{Abstract}

Background To improve treatment outcomes for tuberculosis (TB), efforts to reduce treatment failure are necessary. The aim of our study was to describe the characteristics of subjects who had failed treatment of tuberculosis and identify the risk factors for treatment failure and poor compliance using national data.

Methods A multicenter cross-sectional study was performed on tuberculosis subjects whose final outcome was reported as treatment failure during 2015-2017. The same number of subjects with treatment success during the same study period were randomly selected for comparison. Demographics, microbiological, radiographic, and clinical data were collected based on in-depth interviews by TB nurse specialists at all Public Private Mix (PPM) participating hospitals in South Korea.

Results A total of 52 tuberculosis patients with treatment failure were enrolled. In a multivariable analysis, the presence of diabetes, previous history of tuberculosis, and cavity were identified as risk factors for treatment failure; and Medicaid support was a favorable factor for treatment success (area under the curve [AUC]: 0.79). Age, low body mass index (BMI), presence of diabetes, preexisting lung disease, positive sputum acid-fast bacilli (AFB) smear result, and the presence of multidrug-resistant tuberculosis ( MDR-TB) were significantly associated with presence of cavities. Younger age, lower BMI and previous history of TB were associated with poor compliance during treatment (AUC: 0.76).

Conclusion To reduce treatment failure, careful evaluation of the presence of diabetes, previous TB history, underlying lung disease, cavity, results of sputum AFB smears, and socioeconomic status are needed. To enhance treatment compliance, more attention should be paid to younger patients with lower BMls during follow-up.

\section{Background}

Tuberculosis (TB) remains an unsolved public health problem despite the strenuous efforts exerted by numerous countries (1). In spite of the high economic status and medical services system, South Korea is included as an intermediate TB burden country and is in first place for TB development among the countries that are members of the Organization for Economic Co-operation and Development (2). To overcome this situation, the Public Private Mix (PPM) collaboration model that supports diagnosis and treatment of TB has been implemented as a national TB control strategy, and as a consequence, the rate of TB has been decreasing. However, a considerable rate of treatment failure still exists, complicated by the development of drug resistance, and disease-related morbidities and mortalities that prevent the curing of TB. To triumph in battling tuberculosis, further efforts to decrease poor outcomes such as treatment failure and increase treatment compliance are needed. The clinical characteristics of those who had failed treatment are limited because of difficulties in collecting appropriate subjects. In previous studies, human immunodeficiency virus (HIV) co-infection, previous history of TB, sputum smear positivity after two months of treatment, male sex, young or advanced age, drug resistance, and residence 
in a solitary area have been proposed as risk factors for poor outcome (3-9). However, such studies were performed on a small number of patients in high-TB burden countries with limited medical resources. Because South Korea has a different socioeconomic environment, a low rate of HIV infection, and high access to medical services $(10,11)$, a different strategy to control TB is required in South Korea.

The aim of our study was to describe the subjects' characteristics and to identify the risk factors of treatment failure and poor compliance for the purpose of predicting risk groups to improve treatment outcomes. Collecting enough treatment failure cases from individual institutions was difficult; therefore, we collected national data of treatment failure cases from all the PPM-participating hospitals.

\section{Methods}

In South Korea, physicians must notify the diagnosis and treatment of TB when they initially diagnose or suspect TB and multidrug-resistant tuberculosis (MDR-TB). Under the PPM project, all patients are followed during treatment until the report of final treatment outcomes by TB nurse specialists dispatched to private PPM hospitals. TB nurse specialists record information about medications, side effects, and compliance with the Korean National TB Surveillance System of PPM hospitals (12). More than 210 TB nurse specialists at 127 PPM hospitals and 236 public health officials at 254 public health centers across the country work under the PPM projects. Approximately $69 \%$ of new TB patients were treated at PPM hospitals in 2017. From January 2015 to December 2017, data of subjects who failed TB treatment were collected from all the PPM participating hospitals and TB nurse specialists at each hospital completed treatment failure case report forms. Treatment failure was defined as remaining culture-positive after four months of treatment or at the end of treatment (13). Patients who took at least $95 \%$ of medication as prescribed by the clinician were defined to be compliance group (14). Baseline characteristics such as age, sex, body mass index (BMI), respiratory symptoms, previous history of TB, co-existing comorbidities, and smoking and alcohol history were recorded. Furthermore, results of radiographic, microbiological, and clinical data, including sputum smear, drug susceptibility test, treatment regimen, and treatment compliance, were retrospectively collected by TB nurse specialists. Presence or absence of cavity were judged by the results of chest X-ray or chest computed tomography. After collecting information about treatment failure cases, the same number of subjects with treatment success during the same study period were randomly selected and the characteristics of these two groups were compared. Additionally, the characteristics of treatment compliance and noncompliance groups were also compared. Based on the results, a prediction model for treatment failure was constructed.

\section{Statistical analysis}

The subjects' characteristics were presented as the mean and standard deviation for continuous variables and as relative frequencies for categorical variables. Statistical analysis was performed using $R$ (version 3.6.0). Means were compared using a t-test or analysis of variance (ANOVA), and categorical variables were compared using a chi-squared test or Fisher's exact test. To construct early prediction model, logistic regression was performed including significant variables in univariable analysis at $P$ value 
$<0.1$ in addition to age, sex, and BMl; variables that could be measured at the beginning of TB treatment such as respiratory symptoms, previous history of TB, social history, comorbidities, radiographic characteristic, and sputum acid-fast bacilli (AFB) smear results were included. The best model was selected with the stepwise selection method using stepAIC function in MASS package. To compare the classification ability of each model, the area under the curve (AUC) of the receiver operating characteristic curve (ROC) was calculated using the ROCR package. To assess predictive validity, leave-one-out cross validation (LOOCV) using boot package was performed.

\section{Ethical approval}

The study was conducted in accordance with the Declaration of Helsinki. The Korea Centers for Disease Control and Prevention (KCDC) has the authority to hold and analyze surveillance data for public health and research purposes. KCDC approved the data use and provided data without personal identification information.

\section{Results}

\section{Baseline characteristics}

A total of 52 subjects who failed treatment and 50 who had treatment success during the study period were enrolled. The demographic and clinical characteristics of these participants are summarized in Table 1. The mean age was 44.5 years, and 38 (73.1\%) were men. Among them, 29 (55.8\%) were newly diagnosed cases, $12(23.1 \%)$ recurred cases, 7 (13.5\%) re-treated cases after failure, and $3(5.8 \%)$ retreated cases after treatment cessation. Twenty-seven (51.9\%) were AFB smear-positive. The initial treatment regimen was as follows: HREZ was administered to 40 (76.9\%) patients, HRE was administered to $1(1.9 \%)$ patient, and $11(21.2 \%)$ patients were administered others. The demographic and clinical characteristics of the randomly selected subjects who succeeded during the same study period, are also summarized in Table 1. Age, sex, BMI, and smoking and alcohol history were not significantly different between the two groups. However, the presence of diabetes mellitus, previous history of TB, and sputum AFB smear positivity were increased in the treatment failure group. In addition, the cavity was found more frequently on chest radiography in the treatment failure group. Compared to subjects without cavities, those with cavity presented with more underlying lung disease, diabetes, and positive sputum AFB smear results. Detailed characteristics comparing these groups are summarized in Supplemental Table S1. Nineteen (36.5\%) subjects in the treatment failure group had MDR-TB. Fifty (28.8\%) subjects presented non-compliance during treatment. A comparison of the characteristics between the treatment compliant and noncompliant groups is summarized in Supplemental Table S2. Noncompliant subjects were younger $(P=0.02)$ and less obese $(P=0.02)$ than compliant subjects. A previous history of TB was less frequent in the noncompliant group. There was no association between age and BMI in both sex $(P=0.12$, Supplemental Figure 51$)$. The relative distribution of overlaps among subjects who failed treatment, presented with MDR-TB, and were noncompliant are summarized in Supplemental Figure S2. 
For multivariable analysis, variables of age, sex, presence of diabetes, previous history of TB, Medicaid support, presence of cavity, and positivity of sputum AFB smear were included; the presence of diabetes mellitus, previous history of TB, presence of cavity, and absence of Medicaid support were selected as independent risk factors for treatment failure in all study subjects (Table 2). The AUC of the ROC of this model was 0.792 (Figure 1A). The predictive validity using LOOCV of this model was 0.795 . Younger age, lower BMI, presence of diabetes, presence of preexisting lung disease, positivity of sputum AFB smear, and presence of MDR-TB were significantly associated with the presence of cavities (Table 2). In subjects without MDR-TB, the presence of diabetes (odds ratio [OR] $=3.58,95 \%$ confidence interval [CI]: 0.9713.23), previous history of TB ( $O R=3.45,95 \% \mathrm{Cl}: 1.39-8.54)$, and presence of cavity $(\mathrm{OR}=2.74,95 \% \mathrm{Cl}$ : 0.90-8.36) were selected for predicting treatment failure, and the AUC of the ROC curve for this model was 0.73 (Supplemental Figure S3). For compliance during treatment, variables of age, sex, BMI, and previous history of TB were included for multivariable analysis. Younger age, lower BMI, and previous history of TB were associated with poor drug compliance, and the AUC of the ROC curve for this model was 0.765 (Figure 1B). The predictive validity of this model was 0.887 .

\section{Discussion}

In our study, we compared the characteristics of patients with treatment failure with those of treatment success and built a prediction model for treatment failure with high predictive power. The presence of diabetes, previous history of TB, and cavity were independent risk factors for treatment failure, and Medicaid support was favorable one for treatment success. For the presence of cavities, younger age, low BMI, diabetes, preexisting lung disease, positive sputum AFB smear, and MDR-TB were independent risk factors. Since treatment compliance is an essential component of treatment success, younger age, lower $\mathrm{BMI}$, and previous history of TB were unfavorable predictors for compliance, and these predictors were connected to each other acting as complicated effect modifiers.

The first model for predicting treatment failure by Kalhori et al. (15) used clinical data including old age, male sex, body weight, nationality, prisoner status, and previous history of TB, and achieved an AUC of 0.70. Recently, Sauer et al. (16) tried to predict treatment failure by machine learning using demographic and laboratory data and reported a best AUC of 0.74 . However, this model lacked information about comorbidities; our model included such variables and yielded considerably high prediction power, an AUC of 0.79 . Furthermore, our model was constructed based on routinely collected data we recruited retrospectively that were easily gathered in clinical practice.

The remarkable traits to review carefully are the presence of diabetes and age. Diabetes is known to be associated with the development of TB, possibly mediated by several mechanisms of proinflammatory cytokine (17-21), especially if diabetes-related complications co-exist (22). In our study, we identified that diabetes was not only related to the development of TB, but also related to treatment failure and the presence of cavities. Older age is a well-known risk factor for the development of TB and higher TB- 
related death rates $(23,24)$. However, younger age was related to the presence of cavity, and poor compliance to treatment. Furthermore, there have been reports that $\mathrm{BMI}$ is inversely associated with the risk of TB (25). Obesity presented a protective effect, while a lower BMI was associated with the development of TB (26) and higher TB-related mortality (27). However, BMI is also associated with metabolic syndrome such as diabetes mellitus (28), so these opposite effects of BMI could confuse their role in TB (26). In our study population, BMI in subjects with diabetes was not significantly different that in those without diabetes ( 20.75 vs. $22.30 ; P=0.13$ ); instead, lower $B M I$ was related to the presence of cavity and poor compliance to treatment. Medicaid support was associated with more treatment success and ensured the importance of national efforts such as the PPM program in defeating tuberculosis.

Although our study revealed the complex associations of several risk factors, there are limitations that should be noted when interpreting our results. This study is a retrospective case-control study, and data were recruited after results of the sputum culture reports came out, so there could be some missing information for each variable. The problem of recall bias from patients, families, and TB nurse specialists may exist. Though we tried to collect cases, especially focusing on non MDR-TB patients, as a complete enumeration among PPM participating hospital, the number of enrolled patients was small; which reflects the frequency of the treatment failure in South Korea. Post hoc power analysis estimates power of our study as 0.767 if we assume medium to large effect size of 0.45 . However, further large prospective cohort studies to confirm our findings are necessary. Additionally, these cases were recruited from PPM participating hospitals, and although approximately $70 \%$ of TB patients are treated under the PPM program, this could limit the generalizations of our study.

\section{Conclusions}

In conclusion, in order to reduce treatment failure, the presence of diabetes, previous history of TB, underlying lung disease, sputum AFB smear results, and socioeconomic status should be carefully evaluated, and more attention has to be given to younger patients with lower BMls during follow-up to improve treatment compliance. Further, larger studies are needed in order to confirm our findings.

\section{List Of Abbreviations}

AFB, acid-fast bacilli; BMI, body mass index; $\mathrm{Cl}$, confidence interval; MDR-TB, multidrug-resistant tuberculosis; OR, odds ratio; PPM, Public Private Mix; ROC, receiver operating characteristic curve; TB, tuberculosis; AUC, under the curve

\section{Declarations}

\section{Declarations}

Not applicable

\section{Ethics approval and consent to participate}


The Korea Centers for Disease Control and Prevention (KCDC) has the authority to hold and analyze surveillance data for public health and research purposes. KCDC approved the data use and provided data without personal identification information.

\section{Consent for publication}

Not applicable

\section{Availability of data and materials}

The ownership of the primary dataset lies with the Korea Centers for Disease Control and Prevention $(K C D C)$. The datasets used and/or analyzed during the present study are available on reasonable request after obtaining permission from the KCDC in advance.

\section{Competing interests}

All authors declare that they have no competing interest

\section{Funding}

There was no funding from agencies in the public, commercial, or not-for-profit sectors.

\section{Authors' contributions}

JSK, HKK, JM, HWK, JL, JSP, and SSL conceived of the analysis. HKK performed all analyses. SSL, JSK, and JSP supervised the research project. HKK and SSL drafted the manuscript. MJ, HWK, JL, JSK, and JSP provided a substantive review and edited the manuscript. All authors have read and approved the final manuscript.

\section{Acknowledgements}

The authors thank TB specialist nurses across the country who interviewed patients and their families.

\section{References}

1. World Health Organization. Global Tuberculosis Report. Geneva: WHO; 2018. https://www.who.int/tb/publications/global_report/en/. Accessed 18 June 2019

2. Go U, Park M, Kim U-N, Lee S, Han S, Lee J, Yang J, Kim J, Park S, Kim Y, et al. Tuberculosis prevention and care in Korea: evolution of policy and practice. J Clin Tuberc Other Mycobact Dis. 2018; $11: 28-36$

3. Mekonnen D, Derbie A, Desalegn E. TB/HIV co-infections and associated factors among patients on directly observed treatment short course in Northeastern Ethiopia: a 4 years retrospective study. BMC Res Notes. 2015;8(1):666 
4. Tesfahuneygn G, Medhin G, Legesse M. Adherence to Anti-tuberculosis treatment and treatment outcomes among tuberculosis patients in Alamata District, northeast Ethiopia. BMC Res Notes. 2015; 8:503

5. SaE Grzybowski. The fate of cases of pulmonary tuberculosis under various treatment programmes. Bull Int Union Tuberc. 1978; 53:70-5

6. Munoz-Sellart M, Cuevas LE, Tumato M, Merid Y, Yassin MA. Factors associated with poor tuberculosis treatment outcome in the Southern Region of Ethiopia. Int J Tuberc Lung Dis. 2010;14(8):973.9

7. Amante TD, Ahemed TA. Risk factors for unsuccessful tuberculosis treatment outcome (failure, default and death) in public health institutions, Eastern Ethiopia. Pan Afr Med J. 2015; 20:247

8. World Health Organization. Adherence to long-term therapies: evidence for action. Geneva: WHO; 2003:33-7

9. Gebrezgabiher G, Romha G, Ejeta E, Asebe G, Zemene E, Ameni G. Treatment outcome of tuberculosis patients under directly observed treatment short course and factors affecting outcome in southern Ethiopia: a five-year retrospective study. PLoS ONE. 2016;11(2): e0150560

10. Lee YH, Bang JH, Park SM, Kang CR, Cho S-I, M-d O, Lee J-K. Costeffectiveness of voluntary HIV testing strategies in a very low-prevalence country, the Republic of Korea. J Korean Med Sci. 2018; 33: e304

11. Suguimoto SP, Techasrivichien T, Musumari PM, El-saaidi C, Lukhele BW, Ono-Kihara M, Kihara M. Changing patterns of HIV epidemic in 30 years in East Asia. Current HIV/AIDS Rep. 2014; 11: 134-45

12. Kang H-Y, Yoo H, Park W, Go U, Jeong E, Jung K-S, Son H. Tuberculosis notification completeness and timeliness in the Republic of Korea during 2012-2014. Osong Public Health Res Perspect. 2016; 7: $320-6$

13. WHO revised definitions and reporting framework for tuberculosis. Eurosurveillance editorial team. Euro Surveill. 2013 Apr 18;18(16):20455

14. Tesfahuneygn G, Medhin G, Legesse M. Adherence to Anti-tuberculosis treatment and treatment outcomes among tuberculosis patients in Alamata District, northeast Ethiopia. BMC Res Notes. 2015; 8: 503

15. Sharareh R. Niakan Kalhori MN, Zeng Xiao-Jun. A Logistic Regression Model to Predict High Risk Patients to Fail in Tuberculosis Treatment Course Completion. International Journal of Applied Mathematics. 2008; 40:2

16. Sauer CM, Sasson D, Paik KE, McCague N, Celi LA, Sánchez Fernández I, Illigens BMW. Feature selection and prediction of treatment failure in tuberculosis. PLoS One. 2018;13(11): e0207491

17. Saiki O, Negoro S,Tsuyuguchi I, YamamuraY.Depressed immunological defence mechanisms in mice with experimentally induced diabetes. Infect Immun 1980; 28:127-31

18. Al-Attiyah RJ, Mustafa AS. Mycobacterial antigen-induced T helper type 1 (Th1) and Th2 reactivity of peripheral blood mononuclear cells from diabetic and non-diabetic tuberculosis patients and 
Mycobacterium bovis bacilli Calmette-Guerin (BCG)-vaccinated healthy subjects. Clin Exp Immunol 2009; 158:64-73

19. Martens GW, Arikan MC, Lee J, Ren F, Greiner D, Kornfeld H. Tuberculosis susceptibility of diabetic mice. Am J Respir Cell Mol Biol 2007; 37:518-24.

20. Restrepo BI, Fisher-Hoch SP, Pino PA, et al. Tuberculosis in poorly controlled type 2 diabetes: altered cytokine expression in peripheral white blood cells. Clin Infect Dis 2008; 47:634-41

21. Vallerskog T, Martens GW, Kornfeld H. Diabetic mice display a delayed adaptive immune response to Mycobacterium tuberculosis. J Immunol 2010; 184:6275-82

22. Meghan A Baker, Hsien-Ho Lin, Hsing-Yi Chang, Megan B Murray. The Risk of Tuberculosis Disease Among Persons With Diabetes Mellitus: A Prospective Cohort Study. Clin Infect Dis. 2012 Mar;54(6):818-25

23. S Rajagopalan. Tuberculosis and Aging: A Global Health Problem. Clin Infect Dis. 2001;33(7):1034-9.

24. P D Davies. Tuberculosis in the Elderly. Epidemiology and Optimal Management. Drugs Aging. 1996;8(6):436-44

25. Lönnroth K, Williams BG, Cegielski P, Dye C. A consistent log-linear relationship between tuberculosis incidence and body mass index. Int J Epidemiol 2010; 39:149-55

26. Hsien-Ho Lin, Chieh-Yin Wu, Chih-Hui Wang, Han Fu, Knut Lönnroth, Yi-Cheng Chang, Yen-Tsung Huang. Association of Obesity, Diabetes, and Risk of Tuberculosis: Two Population-Based Cohorts. Clin Infect Dis 2018 Feb 10;66(5):699-705

27. Jinsoo Min, Ju Sang Kim, Hyung Woo Kim, Ah Young Shin, Hyeon-Kyoung Koo, Sung-Soon Lee, Yang-Ki Kim, Kyeong-Cheol Shin, Jung Hyun Chang, Gayoung Chun, Joosun Lee, Mi Sun Park, Jae Seuk Park. Clinical Profiles of Early and Tuberculosis-Related Mortality in South Korea Between 2015 and 2017: A Cross-Sectional Study. BMC Infect Dis 2019 Aug 22;19(1):735

28. Atilla Engin. The Definition and Prevalence of Obesity and Metabolic Syndrome. Adv Exp Med Biol 2017; 960:1-17

\section{Tables}

Table 1. Clinical characteristics comparing treatment success group and treatment failure group 


\begin{tabular}{|c|c|c|c|}
\hline & $\begin{array}{l}\text { Treatment success } \\
\qquad(\mathrm{N}=50)\end{array}$ & $\begin{array}{l}\text { Treatment failure } \\
\qquad(\mathrm{N}=52)\end{array}$ & P-value \\
\hline$\overline{\text { Age }}$ & $44.0(31.5-65)$ & $44.5(29.5-67)$ & 0.84 \\
\hline Male sex & $28(56.0 \%)$ & $38(73.1 \%)$ & 0.11 \\
\hline BMI & $21.1 \pm 2.88$ & $20.9 \pm 3.07$ & 0.72 \\
\hline Smoking & & & 0.67 \\
\hline Current & $10(20.0 \%)$ & $13(25.0 \%)$ & \\
\hline Ex- & $10(20.0 \%)$ & $7(13.5 \%)$ & \\
\hline Never & $30(60.0 \%)$ & $32(61.5 \%)$ & \\
\hline Drinking & & & 0.21 \\
\hline Heavy & $3(6.0 \%)$ & $3(5.8 \%)$ & \\
\hline Social & $19(38.0 \%)$ & $19(36.5 \%)$ & \\
\hline None & $24(48.0 \%)$ & $30(57.7 \%)$ & \\
\hline Underlying disease & & & \\
\hline Diabetes & $5(10.0 \%)$ & $4(26.9 \%)$ & 0.041 \\
\hline Lung ds & $3(6.0 \%)$ & $4(7.7 \%)$ & $>0.99$ \\
\hline Heart ds & $1(2.0 \%)$ & $3(5.8 \%)$ & 0.24 \\
\hline Liver ds & $2(4.0 \%)$ & $1(1.9 \%)$ & $>0.99$ \\
\hline Kidney ds & $1(2.0 \%)$ & $3(5.8 \%)$ & 0.36 \\
\hline Prev TB Hx & $8(16.0 \%)$ & $19(36.5 \%)$ & 0.01 \\
\hline Symptom & & & \\
\hline Cough/sputum & $20(40.0 \%)$ & $29(55.8 \%)$ & 0.16 \\
\hline Dyspnea & $10(20.0 \%)$ & $7(13.5 \%)$ & 0.43 \\
\hline Chest pain & $7(14.0 \%)$ & $3(5.8 \%)$ & 0.20 \\
\hline Hemoptysis & $1(2.0 \%)$ & $3(5.8 \%)$ & 0.62 \\
\hline Fever & $5(10.0 \%)$ & $3(5.8 \%)$ & 0.48 \\
\hline Weakness & $0(0 \%)$ & $1(1.9 \%)$ & $>0.99$ \\
\hline Weight loss & $6(12.0 \%)$ & $4(7.7 \%)$ & 0.52 \\
\hline Asymptomatic & $17(34.0 \%)$ & $15(28.8 \%)$ & 0.73 \\
\hline Enrollment & & & \\
\hline Newly diagnosed & $42(84.0 \%)$ & $29(55.8 \%)$ & \\
\hline Recurred & $8(16.0 \%)$ & $12(23.1 \%)$ & \\
\hline Retreatment & $0(0 \%)$ & $7(13.5 \%)$ & \\
\hline Default & $0(0 \%)$ & $3(5.8 \%)$ & \\
\hline Social Hx & & & \\
\hline Occupation & $18(36.0 \%)$ & $23(44.2 \%)$ & 0.62 \\
\hline Marriage & $28(56.0 \%)$ & $33(63.5 \%)$ & 0.13 \\
\hline Medicaid & $6(12.0 \%)$ & $1(1.9 \%)$ & 0.06 \\
\hline CPA & & & \\
\hline Normal & $4(8.0 \%)$ & $2(3.8 \%)$ & 0.43 \\
\hline Cavity (+) & $8(17.0 \%)$ & $26(50.0 \%)$ & 0.001 \\
\hline Unilateral & $26(52 \%)$ & $23(44.2 \%)$ & 0.30 \\
\hline
\end{tabular}




\begin{tabular}{|c|c|c|c|c|}
\hline \multicolumn{2}{|c|}{ Bilateral } & \multirow[t]{2}{*}{$17(34 \%)$} & \multicolumn{2}{|l|}{$27(51.9 \%)$} \\
\hline \multicolumn{4}{|c|}{ AFB smear } & \\
\hline & Positive & $11(23.4 \%)$ & $27(51.9 \%)$ & \\
\hline \multicolumn{2}{|l|}{ DST } & \multirow{6}{*}{$\begin{array}{c}46(92.0 \%) \\
2(4.0 \%) \\
1(2.0 \%) \\
1(2.0 \%)\end{array}$} & & $<0.001$ \\
\hline & All sensitive & & $21(40.4 \%)$ & \\
\hline & $\mathrm{R}$ to INH & & $5(9.6 \%)$ & \\
\hline & $\mathrm{R}$ to RFP & & $5(9.6 \%)$ & \\
\hline & Any other $\mathrm{R}$ & & $2(3.8 \%)$ & \\
\hline \multicolumn{2}{|c|}{ Treatment regimen } & & & \\
\hline HREZ & $38(76.0 \%)$ & \multicolumn{2}{|l|}{$40(76.9 \%)$} & \\
\hline $\mathrm{HRE}$ & $11(22.0 \%)$ & \multicolumn{2}{|l|}{$1(1.9 \%)$} & \\
\hline Others & $1(2.0 \%)$ & $11(21.2 \%)$ & & \\
\hline \multicolumn{5}{|c|}{ Drug compliance } \\
\hline & Good compliance & \multirow{2}{*}{$\begin{array}{c}50(100 \%) \\
0(0 \%)\end{array}$} & $37(71.2 \%)$ & \\
\hline & Poor compliance & & $15(28.8 \%)$ & \\
\hline
\end{tabular}

BMI, body mass index; ds, disease; Hx, history; R, resistance; INH, isoniazid; RFP, rifampin; MDR, multi-drug resistance

Table 2. Multivariable analysis for treatment failure, presence of cavity, and treatment compliance

\begin{tabular}{llcc}
\hline & Risk factors & Odds ratio & 95\% CI \\
\hline \hline Treatment failure & Diabetes mellitus & 3.25 & $0.89-11.91$ \\
& Previous history of TB & 2.32 & $0.72-7.41$ \\
\hline & Medicaid & 0.11 & $0.01-1.29$ \\
\hline & Presence of Cavity & 4.78 & $1.72-13.23$ \\
\hline Cavity & Age & 0.957 & $0.92-0.99$ \\
& Body mass index & 0.824 & $0.67-1.01$ \\
\hline \multirow{2}{*}{ Diabetes mellitus } & 3.897 & $0.97-15.59$ & \\
& Pre-existing lung disease & 13.373 & $1.37-130.22$ \\
\hline Poor compliance & Age & 6.730 & $2.17-20.90$ \\
\cline { 2 - 3 } & Sputum smear positive & 6.927 & $1.66-28.84$ \\
\hline & Body mass index & 0.964 & $0.93-0.99$ \\
& Previous history of TB & 0.773 & $0.59-1.00$ \\
\hline
\end{tabular}

TB, tuberculosis; MDR, multi-drug resistance 
Figures
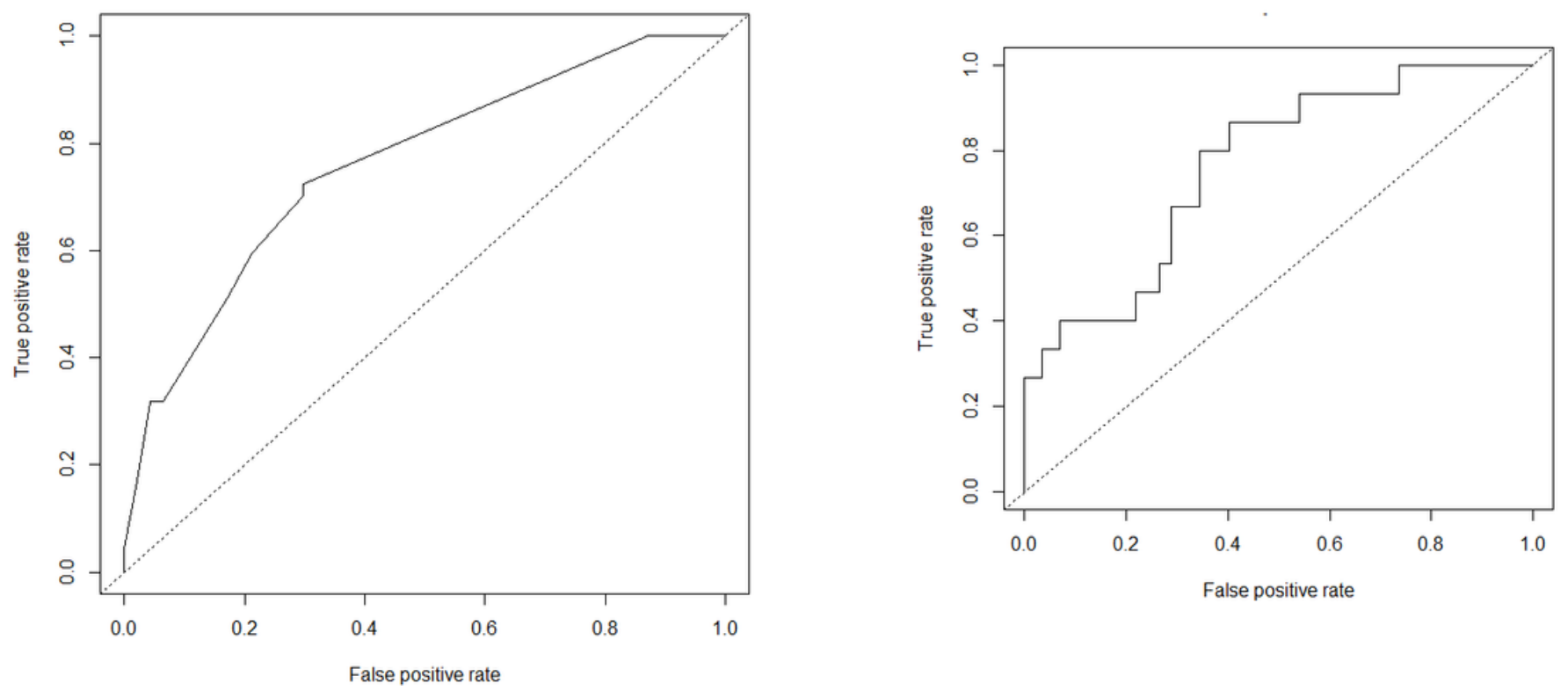

\section{Figure 1}

Receiver operating curve for predicting treatment failure (A) and non-compliance to treatment (B) Figure legends: (A) AUC: 0.79, (B) AUC: 0.76

\section{Supplementary Files}

This is a list of supplementary files associated with this preprint. Click to download.

- Supplementsrevcleared.docx 